\title{
Chronic tonsillar enlargement and cough: preliminary evidence of a novel and treatable cause of chronic cough
}

\author{
S.S. Birring*, C. Passant*, R.B. Patel*, B. Prudon*, G.E. Murty*, I.D. Pavord*
}

Chronic tonsillar enlargement and cough: preliminary evidence of a novel and treatable cause of chronic cough. S.S. Birring, C. Passant, R. B. Patel, B. Prudon, G.E. Murty, I.D. Pavord. C) ERS Journals Ltd 2004.

ABSTRACT: Tonsillar enlargement is sometimes seen in patients with otherwise unexplained chronic cough although its significance is unclear. In this study, the authors set out to test the hypothesis that cough symptoms and cough reflex sensitivity will improve after tonsillectomy in patients with otherwise unexplained chronic cough and enlarged tonsils.

Eight consecutive patients with unexplained chronic cough and enlarged tonsils were recruited from 236 patients seen in a cough clinic between 2000 and 2001 . Six patients with enlarged tonsils and no cough who were undergoing tonsillectomy for other reasons were recruited as a control group. All patients rated cough severity on a cough visual analogue score $(0-100 \mathrm{~mm})$ and had capsaicin cough reflex sensitivity measurement twice before and again 3 months after tonsillectomy.

Patients with a chronic cough had heightened cough reflex sensitivity compared with the control group at baseline. There was a significant improvement in mean cough visual analogue score 3 months after tonsillectomy in patients with chronic cough (mean difference $30 \mathrm{~mm} ; \mathbf{9 5} \%$ confidence interval of difference $8-51 \mathrm{~mm}$ ). The geometric mean concentration of capsaicin required to cause five coughs increased from 4 to $207 \mu \mathrm{mol} \cdot \mathrm{L}^{-1}$ after tonsillectomy in patients with chronic cough (mean difference from baseline 5.6 doubling concentrations; 95\% confidence interval of difference 3.1-8.2). There was no change in cough reflex sensitivity in control patients after tonsillectomy.

These preliminary findings suggest for the first time a possible role for tonsillectomy in patients with enlarged tonsils in whom other causes of cough have been ruled out. Eur Respir J 2004; 23: 199-201.
*Institute for Lung Health, Dept of Respiratory Medicine, Glenfield Hospital, and ${ }^{\#}$ Dept of ENT, Leicester Royal Infirmary, Leicester, UK.

Correspondence: S.S. Birring, Institute for Lung Health, Dept of Respiratory Medicine, Glenfield Hospital, Leicester, LE3 9QP, UK. Fax: 441162367768

E-mail: sb134@le.ac.uk

Keywords: Capsaicin

chronic cough

cough sensitivity

tonsillectomy

tonsillitis

\section{Received: June 122003}

Accepted: September 32003

S.S. Birring is a British Lung Foundation clinical research fellow. This study was funded by the British Lung Foundation and University Hospitals of Leicester NHS Trust.
Cough is one of the commonest causes of presentation to general practice. At any one time $20 \%$ of the UK population have a troublesome cough and sufferers consume 75 million doses of over-the-counter antitussive medication annually $[1,2]$. Most cases are acute and are self-limiting although a significant minority with an isolated chronic cough are referred for a specialist opinion. The cause of a chronic cough can be identified relatively simply in most cases with the majority due to one or more of asthma, eosinophilic bronchitis, gastrooesophageal reflux and rhinitis with post-nasal drip [3, 4]. However, in up to $20 \%$ of patients, the cough remains unexplained even after extensive investigation and treatment trials [5]. These patients suffer considerable physical and psychological morbidity [6].

Cough receptors are located in the larynx and other upper airway structures as well as in the lower airways, and conditions associated with upper airway inflammation are commonly implicated in causing chronic cough [2]. Chronic tonsillar enlargement is a common condition that might potentially be associated with upper airway inflammation and cough. Chronic tonsillar enlargement is not widely accepted as a cause of chronic cough although there is one case report of improvement in cough following lingual tonsillectomy in a child [7]. The current authors tested the hypothesis that chronic tonsillar enlargement is present in some patients with unexplained chronic cough, and that cough symptoms and cough sensitivity improve following surgical removal of tonsils.
Methods

\section{Subjects}

Patients with chronic cough were recruited between 2000 and 2001 from a specialised cough clinic that receives referrals from primary and secondary care largely confined to a population of 970,000 within Leicestershire, UK. Investigations were carried out according to a standardised algorithm [4] with the protocol for investigation and treatment and criteria for accepting diagnosis as previously described [4, 8]. Patients with an isolated chronic cough were enrolled into the study if they had enlarged tonsils on external examination (at least Brodsky grade $2+$ : tonsils, occupying $25-50 \%$ of the airway) [9] and otherwise normal Ear Nose Throat (ENT) findings, and had no objective evidence of alternative causes or had failed to respond to specific treatment directed against a suspected cause. A control group comprising randomly selected patients from the waiting list for routine tonsillectomy for recurrent tonsillitis were also recruited. Written consent was obtained from all patients and the protocol for this study was approved by the Leicestershire Research Ethics Committee.

\section{Protocol and clinical measurements}

All subjects had spirometry measurement and patients with cough rated their cough severity on a previously validated 
100-mm horizontal visual analogue scale [10], with 0 being no cough and 100 being worst cough ever, at baseline (mean of two scores taken 1 week apart) and 3 months after tonsillectomy. Capsaicin cough reflex sensitivity was measured by an operator blinded to patient group at baseline (mean of two readings taken 1 week apart) and 3 months after tonsillectomy at the same time of day using a protocol described previously [11]. Briefly, subjects inhaled doubling concentrations of capsaicin $(0.5-500 \mu \mathrm{M})$ in a sequential order at 1-min intervals via a nebuliser attached to a breath-activated dosimeter delivering $8 \mu \mathrm{L}$. The number of coughs in response to each concentration was counted and recorded. The challenge was stopped when five or more coughs occurred or the highest dose of capsaicin was reached. The concentrations of capsaicin that caused two and five coughs $\left(\mathrm{C}_{2}\right.$ and C5 $\mu \mathrm{mol} \cdot \mathrm{L}^{-1}$ ) were calculated by linear interpolation of the $\log$ dose-response curves. Subjects had tonsillectomy using standard procedures by blunt dissection.

\section{Statistical analysis}

Subject characteristics were described and expressed as means \pm SE. The $\mathrm{C}_{2}$ and $\mathrm{C} 5$ were described as geometric mean $\pm \operatorname{logSEM}$ and compared within patients using a paired t-test and between patients using an unpaired t-test. The study had a $80 \%$ power to show a one doubling concentration change in $\mathrm{C} 2$ at the $95 \%$ significance level.

\section{Results}

A total of 236 patients with chronic cough were seen. The primary causes of cough were: cough variant asthma 39 $(17 \%)$, gastro-oesophageal reflux $35(15 \%)$, rhinitis $29(12 \%)$, eosinophilic bronchitis $17(7 \%)$, idiopathic $62(26 \%)$, postviral $17(7 \%)$, bronchiectasis $14(6 \%)$, chronic bronchitis 10 $(4 \%)$, pulmonary fibrosis seven $(3 \%)$, angiotensin converting enzyme inhibitor-related cough four $(2 \%)$, sarcoidosis one $(0.5 \%)$ and lung cancer one $(0.5 \%)$. No patients with an explained chronic cough had evidence of tonsillar enlargement. Amongst the 62 patients with unexplained chronic cough, eight had clinical evidence of tonsillar enlargement. The subject characteristics are shown in table 1 . None of these patients reported symptoms suggesting recurrent tonsillitis. The severity of cough improved in all chronic cough patients after tonsillectomy. The mean \pm SEM cough visual analogue score after tonsillectomy was $27 \pm 12 \mathrm{~mm}$ compared with $57 \pm 11 \mathrm{~mm}$ at baseline (mean difference $30 \mathrm{~mm} ; 95 \%$ confidence interval of difference $8-51 \mathrm{~mm} ; \mathrm{p}<0.02$ ). Patients with a chronic cough had heightened cough reflex sensitivity

Table 1. - Subject characteristics

\begin{tabular}{lcc}
\hline & Controls & Chronic cough \\
\hline Number & 6 & 8 \\
Male \% & $1 \pm 17$ & $2 \pm 25$ \\
Age yrs & $27 \pm 3$ & $37 \pm 5$ \\
Duration of cough yrs & & $7 \pm 5$ \\
FEV1 \% pred & $100 \pm 2$ & $105 \pm 6$ \\
Smoking & & \\
$\quad$ Never & 5 & 8 \\
Ex & 0 & 0 \\
Current & 1 & 0 \\
\hline
\end{tabular}

Data are presented as mean \pm SEM. FEV1: forced expiratory volume in one second. compared with the control group at baseline for both geometric mean $\mathrm{C}_{2}(\mathrm{p}<0.04)$ and $\mathrm{C} 5(\mathrm{p}<0.007$; fig. 1). Cough reflex sensitivity improved significantly after tonsillectomy in patients with chronic cough for both $\mathrm{C}_{2}$ (geometric mean concentration 28.8 versus $1.3 \mu \mathrm{mol} \cdot \mathrm{L}^{-1}$; mean difference from baseline 4.5 doubling concentrations; $95 \%$ confidence interval of difference 1.5-7.4 doubling concentrations; $\mathrm{p}<0.02$ ) and $\mathrm{C} 5$ (geometric mean concentration 207.0 versus $4.1 \mu \mathrm{mol} \cdot \mathrm{L}^{-1}$; mean difference from baseline 5.6 doubling concentrations; $95 \%$ confidence interval of difference $3.1-8.2 ; \mathrm{p}<0.003$ ). There was no significant change in cough reflex sensitivity following tonsillectomy in the control group (fig. 1). There was a significant difference in change in $\mathrm{C} 5(\mathrm{p}<0.005)$ but not $\mathrm{C} 2(\mathrm{p}<0.09)$ between controls and patients following tonsillectomy.

\section{Discussion}

For the first time, it has been shown that chronic tonsillar enlargement is present in some patients with otherwise unexplained chronic cough, and that cough symptoms and cough reflex sensitivity improve 3 months after tonsillectomy. The control population had normal cough sensitivity before intervention and no change in cough sensitivity after. This suggests that there is a functionally important difference in the nature or extent of tonsillar pathology or in the patients'
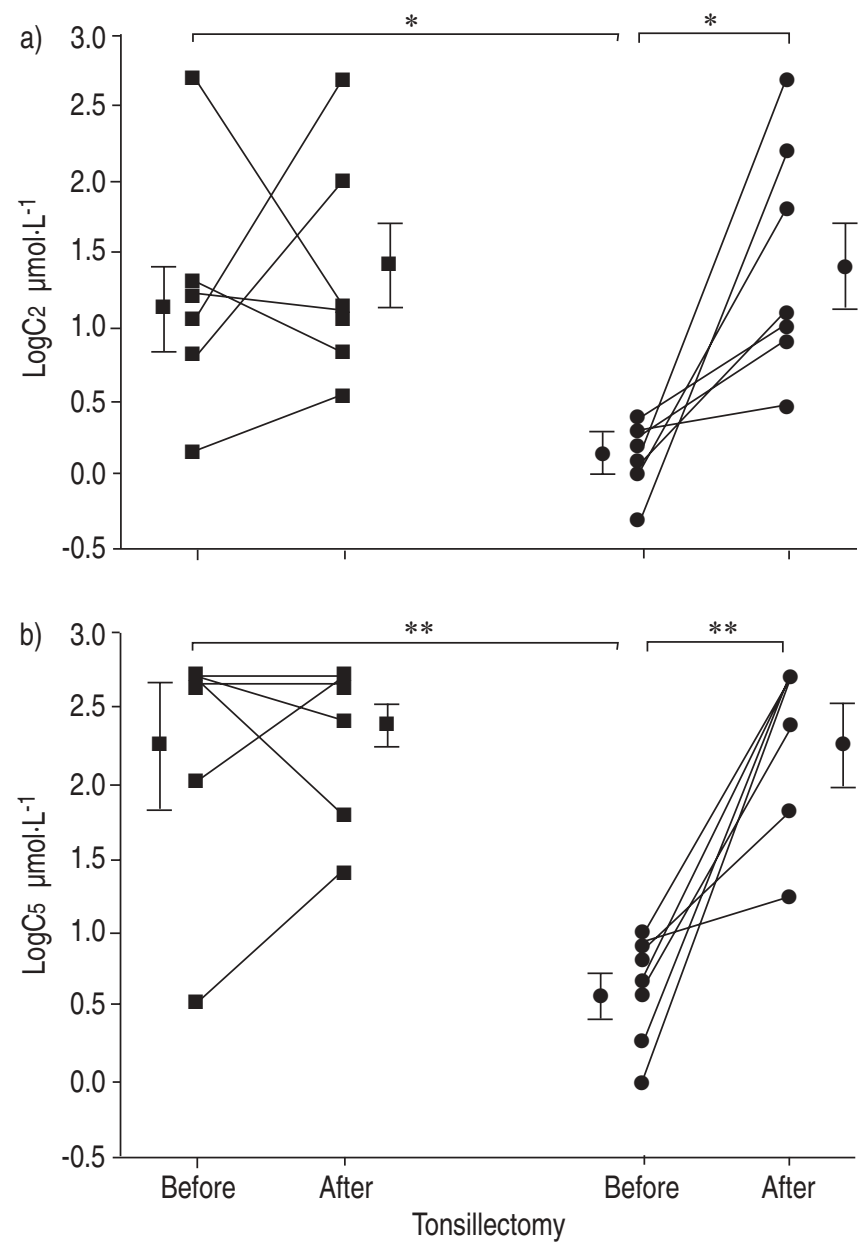

Fig. 1.- Individual log concentrations of capsaicin that caused two and five coughs ( $\mathrm{C}_{2}$ and $\left.\mathrm{C}_{5}\right)$ before and after tonsillectomy in controls ( $)$ and chronic cough subjects $(\bullet)$. Before values represent mean of two observations. Bars represent mean \pm SEM. *: $\mathrm{p}<0.05 ; * *: \mathrm{p}<0.01$. 
response to it. Further studies particularly investigating the pathophysiology of the tonsillar enlargement and its interaction with the cough reflex are required.

As with almost all surgical trials, the present observations are unblinded, uncontrolled and based on small numbers. However, the measurements were made blind to clinical characteristics and the pre-surgery cough reflex sensitivity was assessed on two occasions. Furthermore, the effects of tonsillectomy on symptoms and cough reflex sensitivity were large, well outside the limits of repeatability of the test [10] and considerably greater than the effects of inhaled corticosteroids on cough sensitivity in eosinophilic bronchitis [11]. These factors make it unlikely that the effect seen was due to spontaneous improvement, bias or regression towards the mean. Nevertheless, larger double-blinded studies, ideally incorporating a broader range of objective markers of cough severity, are required before these findings can be regarded as definitive. One further limitation of the current study is that only one assessment was done after tonsillectomy and, although a marked decrease in cough symptoms and cough sensitivity was found, the duration of the effect is not totally clear. Further studies are required to investigate this.

In conclusion, the findings of this study suggest that chronic tonsillar enlargement is a relatively common and treatable cause of otherwise unexplained chronic cough. The findings here emphasise the importance of a careful oropharyngeal examination in the clinical evaluation of chronic cough.

Acknowledgements. The authors would like to thank the Dept of Respiratory Physiology for lung function tests and respiratory out-patient staff for their assistance in follow-up of patients.

\section{References}

1. Morice AH. Epidemiology of cough. Pulm Pharmacol Ther 2002; 15: 253-259.

2. Fuller RW, Jackson DM. Physiology and treatment of cough. Thorax 1990; 45: 425-430.

3. Irwin RS, Madison JM. The diagnosis and treatment of cough. N Engl J Med 2000; 343: 1715-1721.

4. Brightling CE, Ward R, Goh KL, Wardlaw AJ, Pavord ID. Eosinophilic bronchitis is an important cause of chronic cough. Am J Respir Crit Care Med 1999; 160: 406-410.

5. McGarvey LP, Heaney LG, Lawson JT, et al. Evaluation and outcome of patients with chronic non-productive cough using a comprehensive diagnostic protocol. Thorax 1998; 53: 738-743.

6. Birring SS, Prudon B, Carr AJ, Singh SJ, Morgan MD, Pavord ID. Development of a symptom specific health status measure for patients with chronic cough: Leicester Cough Questionnaire (LCQ). Thorax 2003; 58: 339-343.

7. Lewis M, McClay JE, Schochet P. Lingual tonsillectomy for refractory paroxysmal cough. Int $J$ Pediatr Otorhinolaryngol 2000; 53: 63-66.

8. Irwin RS, Boulet LP, Cloutier MM, et al. Managing cough as a defense mechanism and as a symptom. A consensus panel report of the American College of Chest Physicians. Chest 1998; 114: 133S-181S.

9. Brodsky L. Modern assessment of tonsils and adenoids. Pediatr Clin North Am 1989; 36: 1551-1569.

10. Brightling CE, Monterio W, Green RH, et al. Induced sputum and other outcome measures in chronic obstructive pulmonary disease: safety and repeatability. Respir Med 2001; 95: 999-1002.

11. Brightling CE, Ward R, Wardlaw AJ, Pavord ID. Airway inflammation, airway responsiveness and cough before and after inhaled budesonide in patients with eosinophilic bronchitis. Eur Respir J 2000; 15: 682-686. 